\title{
Changes in Language Style and Topics in an Online Eating Disorder Community at the Beginning of the COVID-19 Pandemic: Observational Study
}

Johannes Feldhege ${ }^{1}$, MSc; Markus Moessner ${ }^{1}, \mathrm{PhD}$; Markus Wolf ${ }^{2}, \mathrm{PhD}$; Stephanie Bauer ${ }^{1}, \mathrm{PhD}$

${ }^{1}$ Center for Psychotherapy Research, Heidelberg University Hospital, Heidelberg, Germany

${ }^{2}$ Department of Psychology, University of Zurich, Zurich, Switzerland

Corresponding Author:

Johannes Feldhege, MSc

Center for Psychotherapy Research

Heidelberg University Hospital

Bergheimer Strasse 54

Heidelberg, 69115

Germany

Phone: 49622156 ext 7876

Fax: 496221567350

Email: johannes.feldhege@med.uni-heidelberg.de

\section{Abstract}

Background: COVID-19 has affected individuals with lived experience of eating disorders (EDs), with many reporting higher psychological distress, higher prevalence of ED symptoms, and compensatory behaviors. The COVID-19 pandemic and the health and safety measures taken to contain its spread also disrupted routines and reduced access to familiar coping mechanisms, social support networks, and health care services. Social media and the ED communities on social media platforms have been an important source of support for individuals with EDs in the past. So far, it is unknown how discussions in online ED communities changed as offline support networks were disrupted and people spent more time at home in the first months of the COVID-19 pandemic.

Objective: The aim of this study is to identify changes in language content and style in an online ED community during the initial onset of the COVID-19 pandemic.

Methods: We extracted posts and their comments from the ED community on the social media website Reddit and concatenated them to comment threads. To analyze these threads, we applied top-down and bottom-up language analysis methods based on topic modeling with latent Dirichlet allocation and 13 indicators from the Linguistic Inquiry and Word Count program, respectively. Threads were split into prepandemic (before March 11, 2020) and midpandemic (after March 11, 2020) groups. Standardized mean differences were calculated to estimate change between pre- and midpandemic threads.

Results: A total of 17,715 threads ( $n=8772,49.5 \%$ prepandemic threads; $n=8943,50.5 \%$ midpandemic threads) were extracted from the ED community and analyzed. The final topic model contained 21 topics. CIs excluding zero were found for standardized mean differences of 15 topics and 9 Linguistic Inquiry and Word Count categories covering themes such as ED symptoms, mental health, treatment for EDs, cognitive processing, social life, and emotions.

Conclusions: Although we observed a reduction in discussions about ED symptoms, an increase in mental health and treatment-related topics was observed at the same time. This points to a change in the focus of the ED community from promoting potentially harmful weight loss methods to bringing attention to mental health and treatments for EDs. These results together with heightened cognitive processing, increased social references, and reduced inhibition of negative emotions detected in discussions indicate a shift in the ED community toward a pro-recovery orientation.

(J Med Internet Res 2021;23(7):e28346) doi: $\underline{10.2196 / 28346}$

\section{KEYWORDS}

COVID-19; eating disorders; online eating disorder community; language; mental health; social media; LIWC; Linguistic Inquiry and Word Count; Reddit; topic modeling 


\section{Introduction}

COVID-19, caused by SARS-CoV-2, emerged in late 2019 in Wuhan, China and has since spread worldwide before being declared a global pandemic on March 11, 2020, by the World Health Organization. With the number of infections and deaths in the millions, the COVID-19 pandemic has already led to suffering for much of the world population. The pandemic has also contributed to elevated levels of anxiety, depression, and stress in the general population [1,2], with potentially greater effects on individuals with pre-existing mental disorders [3]. Quarantines, social distancing, lockdowns, and other public health measures taken to contain the spread of COVID-19 also have the potential for adverse effects on psychological well-being [4]. These measures were associated with increased depression, anxiety, and psychological distress in the general population [5], with indications for persisting effects after lockdowns were lifted [6]. During previous epidemics, experiences of quarantine led to long-term increases in depressive symptoms [7] and heightened anxiety symptoms, especially in those with a history of mental disorders [8]. Similarly, the COVID-19 pandemic and its associated public health measures impact individuals with lived experience of eating disorders (EDs) in numerous ways, affecting their symptomatology, social support, coping mechanisms, treatment, and engagement with media and the internet.

Individuals with EDs showed higher psychological distress including heightened fear and health anxiety since the beginning of the pandemic $[9,10]$. ED symptoms such as bingeing and food restriction have worsened [10-13], which has been linked to increased food insecurity, as opportunities to shop for food have been reduced and food shortages appeared in supermarkets [14]. Former patients with bulimia nervosa reported higher shape, weight, and eating concerns; body dissatisfaction; and drive for thinness [13]. Studies have also found a higher prevalence of compensatory behaviors such as purging, excessive exercise, or the abuse of laxative and diuretics during this time $[9,10,12,13]$.

Health and safety measures due to COVID-19, such as quarantine, social distancing, or lockdowns disrupted the structure and routine of everyday life, as most people spent a lot more time at home than usual [15]. For many individuals with EDs, this has led to the loss of familiar coping mechanisms and increased rumination about food, weight, or exercise [11]. Similarly, social support has been impacted by health and safety measures that left many individuals isolated and cut off from their usual social support networks [10]. Additionally, access to health care services and ED treatment has been limited during this time $[13,15,16]$. Although many services transitioned to telehealth applications rather quickly, patients report that the quality of their treatment has declined during the pandemic [10]. At the same time, contacts to helplines and instant chats have increased compared to previous years with individuals contacting these services being more strongly affected by EDs, depression, or anxiety [17].

The COVID-19 pandemic also put topics such as food, weight gain, and physical exercise, which can be triggering for individuals with EDs, into the spotlight of traditional and social media [9,11]. This includes news coverage of food shortages and news reports about potential weight gain due to lower activity levels and increased time spent at home as well as a spread of online workout videos on social media $[14,18]$.

For individuals with mental health issues, and especially for individuals with a lived experience of an ED, or those who are at risk for developing an ED, social media has been an important source of communication and social support from like-minded peers due to the shame and stigma associated with these conditions [19]. The expectation would be that social media became even more important for these individuals during the COVID-19 pandemic, a time when access to other sources of support is reduced and people have to spend more time at home. A mixed-methods study on the impact of the COVID-19 pandemic on individuals with lived experience of an ED in the United Kingdom reported that most participants are spending more time on the internet and on social media and that many felt that this had a negative effect on their ED symptoms [11]. One reason for the negative impact of time spent online might be that some participants visited potentially harmful online pro-ED communities, also called pro-ana (short for pro-anorexia nervosa) or pro-mia (short for pro-bulimia nervosa). These websites or social media channels are considered harmful because they often glorify EDs and encourage visitors to engage in pathological ED-related behaviors rather than supporting them to seek help or recovery from EDs [20,21]. Prominent features in pro-ED communities are "thinspiration" content, text or images that propagate a thin ideal, and weight loss "tips and tricks" $[22,23]$. Effects of visiting pro-ED communities are increases in dieting, body dissatisfaction, negative affect, drive for thinness, and disordered eating behaviors [21,24,25].

Although these communities can be harmful, they can also be a source of social support for individuals who feel that other users with lived experience of EDs can understand them better than real-life friends and family [26,27]. As a consequence, people with EDs, or those at-risk for developing an ED, could be reaching out to these communities during the COVID-19 pandemic to receive the social support they are lacking in their everyday life. A qualitative study on three ED communities on the social media website Reddit during the first months of the COVID-19 pandemic uncovered themes such as increased ED symptomatology, changes in daily routine, and treatment interruptions [28], which echo issues that were also found in survey studies.

Research on social media can complement, extend, and even offer some advantages over more traditional clinical research approaches. For example, on social media, a wide group of individuals is active including those at risk for EDs, those that have never been in treatment, or those that would not be reached by traditional surveys. Furthermore, the analysis of social media text is free from the biases introduced by experimental or interview situations. Social media research has contributed to our understanding of the social and psychological implications of disruptive events such as a global pandemic, for example, through tracking developments and interactions, and analyzing their temporal and geographical distributions [29]. Studying social media can also bring to light how an infodemic, that is, 
health-related misinformation, spreads [30]. A number of phenomena related to the COVID-19 pandemic have already been explored with data from social media websites. One study charted the course of COVID-19 symptoms based on topics and language styles extracted from firsthand accounts infected individuals had shared on Reddit [31]. Another study observed the impact of lockdowns on the language styles of Twitter users in Wuhan and Lombardy [32]. The researchers discovered changes in indicators for cognitive processing, uncertainty, and increased time spent at home.

In this study, we explored how social media activity in an online ED community develops during the first months of the COVID-19 pandemic. Although this time is certainly difficult for all individuals with mental health conditions, it posed a particular challenge for individuals with lived experience of EDs. Recent survey studies have shown not only that these individuals are at high risk of experiencing deteriorating symptomatology but also that they were particularly affected by the health and safety measures imposed to curb the spread of the virus and by the news reports on food shortages, weight gain, and home workouts. We sought to determine whether the aforementioned effects such as worsened ED symptomatology and anxiety and reduction of treatment services and social support are reflected in changes in one of the largest ED communities on the social media website Reddit. Due to lockdowns and quarantines, many individuals were spending more time on the internet and social media, potentially discovering ED communities for the first time or becoming more active in them. These communities differ from other mental health online communities in that they often contain both harmful and supportive elements. Therefore, it is vital to investigate the communities that individuals with lived experience of EDs can encounter on social media and how these communities changed as the COVID-19 pandemic began to develop. To the best of our knowledge, only one qualitative study has investigated online ED communities on the social network Reddit.com at the beginning of the COVID-19 pandemic [28]. In contrast to this study, we took a quantitative approach to one of the largest ED communities on Reddit by using two state-of-the-art quantitative text analysis methods. Such methods allow researchers to turn large samples of texts into quantitative representation and to estimate differences between chosen subsets. Specifically, we applied top-down and bottom-up automated language analysis methods to track verbal behavior during the early weeks of the pandemic. First, we extracted the major topics and estimated changes in their prevalence during this time. Second, by using a validated dictionary approach, we analyzed language styles in this community before and after the initial onset of the pandemic. These two analysis methods each provide distinct insights and at the same time complement each other to produce a richer understanding of the changes in an online ED community during the first months of the COVID-19 pandemic than either method on its own. Because our study focuses on the changes in the community as a whole and not on individual users, the unit of analysis for both methods is a discussion thread, which consists of an initial post and all comments made to this post. The aim of our study was to investigate possible changes in the content and language style of these threads at the beginning of the COVID-19 pandemic.

\section{Methods}

\section{Study Design}

We conducted an observational study in an online ED community to identify changes in content and language style in comment threads after COVID-19 became a global pandemic. We chose March 11, 2020, as the start date for the global pandemic, as it coincides with the declaration of COVID-19 as a global pandemic by the World Health Organization [33]. All data were categorized as prepandemic (before March 11) or midpandemic (after March 11) in a dichotomous variable global pandemic status. All topic and language style variables were $z$ standardized, resulting in variables with a mean of 0 and a SD of 1 , allowing for easier interpretation. Changes in topics and language styles from pre- to midpandemic threads were estimated by subtracting their midpandemic mean standardized prevalence from their prepandemic mean standardized prevalence. These standardized mean differences (SMDs) between pre- and midpandemic prevalences can be considered as analogues to effect sizes such as Cohen $d$. They were illustrated in a graph together with their $99 \%$ CIs. A significant change in the mean prevalence of a topic or language style can be observed if its CI does not include zero. A stricter level of confidence at $99 \%$ was chosen as even small differences can become significant in a large data set such as the one used in this study.

\section{Data Set}

We collected data from a large ED community on the social media website Reddit. The community is not identified by name in this paper, as the users wish to remain anonymous. The community was founded in November 2017 after the largest ED community on Reddit at the time, r/proed, was shut down by the administrators of Reddit for violating its rules (for more information on r/proed, see [34]). Posts and comments in this community were accessed in regular time intervals from April 6 to May 20, 2020, through Reddit's official application programming interface (API) using the R package redditoR. As the API limits the access to 1000 items at a time, posts and comments earlier than April 6 were not available through this approach. To gather earlier posts and comments and, thus, derive a prepandemic sample, we accessed earlier posts and comments in the ED community by users who had contributed at least one post or comment in the time period between April 6 and May 20 . In total, 18,071 posts and 100,143 comments created by 6683 users between November 1, 2019, and May 20, 2020, were available for analysis. We concatenated the text of a post and the texts of all comments made to that post into a single thread, thereby combining texts from different users. This approach was deemed appropriate because the focus of our study was not on changes in individual users but rather on trends in topics and language stylistics in the community as a whole. In the following, threads are used as the unit of analysis. 


\section{Data Preprocessing}

We removed 195 posts and 7 comments made by self-identified bots from the data. The native language of posts and comments was determined using the R packages cld 2 and cld 3 , and 22 comments in a language other than English were excluded from further analyses. The threads were prepared for the text analyses by removing HTML code and Unicode characters.

\section{Topic Modeling}

We used topic modeling with latent Dirichlet allocation (LDA) [35] to discover latent topics in threads. LDA is a state-of-the-art unsupervised bottom-up text analysis method that has previously provided intelligible topics for text corpora from Reddit communities for depression [36] and EDs [34]. It can be applied on large text corpora without manual coding and little input by the researchers. Two additional text preprocessing steps were performed to prepare the threads for topic modeling. First, the removal of numbers, punctuation marks, and stop words, which are common words with little meaning, such as is or this. For this, a list of stop words created by the Snowball stemmer project and included in the $\mathrm{R}$ package tm was used. The second preprocessing step was reducing words to their word stem. The $\mathrm{R}$ package stm was used to estimate a structural topic model of the preprocessed threads. The variable global pandemic status was included as a covariate in the model, allowing the prevalence of topics in threads to vary according to whether they were started before or after the declaration of COVID-19 as a pandemic. An initial search for the appropriate number of topics, $K$, for the corpus was conducted with topic models with different values of $K=3,6,9,12 \ldots 30$. In this first step, the models were evaluated using the indicators exclusivity and semantic coherence to narrow down the number of topics $[37,38]$. In a second step, a number of models with $K=9$ to 21 topics were estimated. For these models, we set the number of runs to 50 for each $\mathrm{K}$, the number of expectation-maximization iterations to a maximum of 200 , and all other parameters to default values. From these candidate models, a final model with $K=21$ topics was chosen based on inspection of semantic coherence and exclusivity and manual evaluation of interpretability of its topics. The exclusivity and semantic coherence for models in both steps are displayed in Figures S1 and S2 in Multimedia Appendix 1. Topics were manually annotated with a topic label by the main author (JF), and topic labels were reviewed by the other authors (MM, MW, and SB). Labels were chosen on the basis of the 15 most characteristic words and 20 most characteristic texts for each topic. The Results section shows the manually chosen topic labels and 7 characteristic words as measured by the FREX metric, which balances how frequent and how exclusive to one topic a word is [37].

\section{Language Style Analysis}

Language style in threads was assessed with a set of 13 indicators that have been shown to be associated with ED-related social online activities [39]. These indicators cover behavioral, affective, social, and cognitive dimensions of language use (see the Results section for the names and exemplary words for the indicators). To assess the frequency of these indicators, we used the Linguistic Inquiry and Word Count (LIWC) text analysis program [40]. LIWC is based on a word count algorithm that searches each text unit for words that are assigned to prespecified language categories in its internal dictionary. Words in a given text are matched to these categories and counted to determine the frequency of each category in the text. We also included the relative frequencies of question marks and exclamation marks provided by LIWC, as their use can be an indicator for complexity reduction in texts [39]. We excluded $161(0.91 \%)$ threads from the analyses because less than $70 \%$ of their words were captured by the LIWC 2015 dictionary to prevent unreliable analyses (eg, short text units or due to misspellings or typing errors).

\section{Results}

The final sample used in the analysis consisted of 17,715 threads $(\mathrm{n}=8772,49.5 \%$ prepandemic threads; $\mathrm{n}=8943,50.5 \%$ midpandemic threads). Descriptive statistics of the threads are listed in Table 1. An average thread contained 4 (SD 7.55) comments, was populated by 4.02 (SD 5.19) users, and consisted of 258.41 (SD 390.18) words. Of the 6554 users that participated in the final sample of threads, 2595 (39.59\% of all users) participated in both pre- and midpandemic threads. These users participated in more threads per day in the midpandemic period (mean 0.12, SD 0.25) than in the prepandemic period (mean 0.08 , SD $\left.0.15 ; t_{2594}=-9.18 ; P<.001\right)$. There were $3215(49.05 \%)$ users who participated in the ED community for the first time during the midpandemic period.

Table 1. Descriptive statistics of comment threads $(\mathrm{N}=17,715)$ in the eating disorder community on the social media website Reddit.

\begin{tabular}{|c|c|c|c|}
\hline Variable & Prepandemic threads & Midpandemic threads & Total \\
\hline Threads, n (\%) & $8772(49.5)$ & $8943(50.5)$ & $17,715(100)$ \\
\hline Threads per day, mean (SD) & $66.45(16.70)$ & $129.61(43.91)$ & $88.13(41.74)$ \\
\hline Users per thread $^{\mathrm{a}}$, mean $(\mathrm{SD})$ & $3.43(3.58)$ & $4.6(6.33)$ & $4.02(5.19)$ \\
\hline Comments per thread, mean (SD) & $3.24(5.20)$ & $4.74(9.24)$ & $4.00(7.55)$ \\
\hline LIWC $^{\mathrm{b}}$ word count per thread, mean (SD) & $204.03(247.54)$ & $311.76(485.53)$ & $258.41(390.18)$ \\
\hline LIWC dictionary words per thread, mean (SD) & $89.83(5.06)$ & $90.51(4.62)$ & $90.17(4.85)$ \\
\hline
\end{tabular}

${ }^{\mathrm{a}}$ Users per thread includes the post author and all users that commented on the thread.

bIWC: Linguistic Inquiry and Word Count. 
The labels of the final topic model, exemplary words for topics and LIWC categories, and their unstandardized mean prevalence rates in threads before and after March 11, 2020, are shown in Table 2. Topics can be subsumed into broad categories such as ED symptom-related topics (binge or restrict, purging, binge foods, low calorie foods); weight, shape, and eating concerns (weight loss or gain, body dysmorphia, exercise, appearance, meals); mental health and treatment (mental health, ED treatment); everyday life (domestic life, entertainment, drinks); social aspects (romantic relationships, social support); EDs in community and society (ED communities, EDs and society); and expressions of emotions (affect).

Overall, the most common topics were affect, support, time, and binge or restrict. The order of topics from most to least prevalent changes from pre- to midpandemic threads, with the topics meals and romantic relationships becoming more common than the topic binge or restrict as an example. SMDs of topics between pre- and midpandemic threads are shown in Figure 1. A total of 15 out of the 21 topics showed a significant change, that is, they had CIs that did not include zero. Significant increases in the first 2 months of the pandemic compared to the prepandemic time period were observed in the prevalence of the following nine topics: affect (SMD 0.079, 99\% CI 0.040-0.117), social support (SMD 0.180, 99\% CI 0.142-0.219), meals (SMD 0.057, 99\% CI 0.019-0.096), romantic relationships (SMD 0.087, 99\% CI 0.048-0.125), mental health (SMD 0.186, 99\% CI 0.148-0.225), ED treatment (SMD 0.049, 99\% CI 0.01-0.088), EDs and society (SMD 0.133, 99\% CI 0.095-0.172), ED community (SMD 0.101, 99\% CI 0.061-0.139), and development of ED (SMD 0.091, 99\% CI
0.052-0.13). The prevalence of the following six topics decreased in midpandemic threads in relation to prepandemic threads: binge or restrict (SMD $-0.186,99 \%$ CI -0.225 to -0.148 ), purging (SMD -0.208, 99\% CI -0.246 to -0.169 ), low calorie foods (SMD -0.048, 99\% CI -0.087 to -0.009 ), drinks (SMD $-0.105,99 \% \mathrm{CI}-0.144$ to -0.067 ), binge foods (SMD $-0.099,99 \% \mathrm{CI}-0.137$ to -0.06 ), and appearance (SMD -0.067, $99 \%$ CI -0.106 to -0.029$)$. Figures of daily mean prevalence rates of all topics between November 1, 2019, and May 20, 2020, can be found in Supplement S3 in Multimedia Appendix 1.

Out of the 13 LIWC categories, 9 showed a significant change, that is, they had a 99\% CI excluding zero. Five LIWC categories, anxiety (SMD 0.086, 99\% CI 0.047-0.125), cognitive processes (SMD 0.18, 99\% CI 0.141-0.218), insight (SMD 0.113, 99\% CI 0.074-0.152), social processes (SMD 0.101, 99\% CI 0.062-0.139), and third-person singular (SMD 0.047, 99\% CI 0.008-0.086), became more frequent in mid- compared to prepandemic threads. Question marks (SMD -0.146, 99\% CI -0.185 to -0.107 ); exclamation marks (SMD -0.043, 99\% CI -0.082 to -0.004 ); and words from the LIWC categories body (SMD $-0.071,99 \% \mathrm{CI}-0.109$ to -0.032 ), health (SMD $-0.041,99 \% \mathrm{CI}-0.079$ to -0.002 ), ingestion (SMD -0.085 , $99 \% \mathrm{CI}-0.124$ to -0.047 ), and death (SMD $-0.055,99 \% \mathrm{CI}$ -0.094 to -0.016 ) were used less frequently in threads after the onset of the COVID-19 pandemic. Figures of daily mean prevalence rates of all LIWC categories between November 1, 2019, and May 20, 2020, can be found in Supplement S4 in Multimedia Appendix 1. 
Table 2. Names, exemplary words, and unstandardized prevalences of topics $(n=21)$ and LIWC categories $(n=15)$ in a corpus of comment threads from the ED community on the social media website Reddit.

\begin{tabular}{|c|c|c|c|}
\hline \multirow[t]{2}{*}{ Name } & \multirow[t]{2}{*}{ Exemplary words ${ }^{\mathrm{a}}$} & \multicolumn{2}{|c|}{ Prevalence in corpus ${ }^{\mathrm{b}}$, mean (SD) } \\
\hline & & Prepandemic threads & Midpandemic threads \\
\hline \multicolumn{4}{|l|}{ Topics } \\
\hline Affect & feel hate brain bad just like els & $11.16(5.33)$ & $11.58(5.49)$ \\
\hline Social support & hope happi deserv thank proud better strong & $7.2(5.67)$ & $8.33(6.69)$ \\
\hline Time & back month ve start ago sinc now & $6.81(4.12)$ & $6.68(4.09)$ \\
\hline Binge/restrict & bing fast tomorrow day restrict urg christma & $6.91(6.60)$ & $5.76(5.68)$ \\
\hline Meals & hungri eat meal food dinner hunger lunch & $5.98(5.33)$ & $6.30(5.87)$ \\
\hline Romantic relationships & friend tell boyfriend said ask told partner & $5.64(5.26)$ & $6.12(5.77)$ \\
\hline Purging & ive im throat cant ur vomit spit & $6.49(6.45)$ & $5.22(5.76)$ \\
\hline Weight loss/gain & gain scale pound lbs lose weight maintain & $5.42(7.55)$ & $5.62(7.62)$ \\
\hline Domestic life & home hous room kitchen money groceri car & $4.21(5.61)$ & $4.26(5.99)$ \\
\hline Mental health & disord anorexia behavior valid mental ed behaviour & $3.80(4.30)$ & $4.66(4.86)$ \\
\hline $\mathrm{ED}^{\mathrm{c}}$ treatment & doctor hospit treatment appoint medic inpati therapist & $3.81(7.15)$ & $4.16(7.23)$ \\
\hline Exercise & burn sleep gym workout faint exercis dizzi & $3.93(5.87)$ & $3.94(6.13)$ \\
\hline Low calorie foods & veggi vegan salad veget soup carrot sauc & $3.84(8.43)$ & $3.45(7.90)$ \\
\hline Body dysmorphia & mirror attract photo skinnier compliment skinni thinner & $3.7(5.71)$ & $3.74(5.79)$ \\
\hline Drinks & drink coffe tea soda coke caffein sweeten & $4.08(9.60)$ & $3.14(8.16)$ \\
\hline Binge foods & cooki chocol peanut cake cream chip pizza & $3.91(7.56)$ & $3.20(6.81)$ \\
\hline EDs and society & peopl cultur judg societi shame agre opinion & $3.07(4.08)$ & $3.66(4.69)$ \\
\hline Entertainment & movi song hair scene mukbang video film & $2.89(6.68)$ & $2.68(6.21)$ \\
\hline Appearance & cloth wear hip boob jean waist shirt & $2.95(7.43)$ & $2.47(6.85)$ \\
\hline ED communities & sub reddit post subreddit pro account delet & $2.15(4.86)$ & $2.69(5.98)$ \\
\hline Development of ED & sister parent grade mom school teacher mum & $2.04(2.99)$ & $2.32(3.29)$ \\
\hline \multicolumn{4}{|l|}{ LIWC $^{d}$ categories } \\
\hline Positive emotion & love, nice, sweet & $3.41(2.70)$ & $3.40(2.54)$ \\
\hline Negative emotion & hurt, ugly, nasty & $3.64(2.66)$ & $3.57(2.33)$ \\
\hline Anxiety & worried, fearful & $0.58(0.95)$ & $0.67(0.94)$ \\
\hline Sadness & crying, grief, sad & $0.87(1.27)$ & $0.83(1.10)$ \\
\hline Cognitive processes & cause, know, ought & $13.45(4.70)$ & $14.26(4.27)$ \\
\hline Insight & think, know & $2.48(1.92)$ & $2.69(1.74)$ \\
\hline Question marks & $?$ & $1.41(3.55)$ & $0.99(1.99)$ \\
\hline Exclamation marks & $!$ & $1.06(2.85)$ & $0.94(2.74)$ \\
\hline Social words & mate, talk, they & $6.30(4.57)$ & $6.76(4.49)$ \\
\hline First-person singular & I, me, mine & $10.08(4.07)$ & $10.01(3.78)$ \\
\hline Third-person singular & she, her, him & $0.59(1.45)$ & $0.66(1.51)$ \\
\hline Body & cheek, hands, spit & $1.41(2.10)$ & $1.28(1.70)$ \\
\hline Health & clinic, flu, pill & $1.67(2.13)$ & $1.60(1.73)$ \\
\hline Ingestion & dish, eat, pizza & $4.18(3.72)$ & $3.88(3.36)$ \\
\hline Death & bury, coffin, kill & $0.12(0.51)$ & $0.10(0.40)$ \\
\hline
\end{tabular}

${ }^{\mathrm{a}}$ For topics, exemplary words are the most characteristic words as measured by the FREX metric [37]. For LIWC categories, exemplary words are taken from the LIWC manual [40]. 
${ }^{\mathrm{b}}$ For topics, prevalence in corpus is measured as the percentage of each topic in the corpus. For LIWC categories, it represents the relative percentage of category words per thread.

${ }^{c} \mathrm{ED}$ : eating disorder

${ }^{\mathrm{d}}$ LIWC: Linguistic Inquiry and Word Count.

Figure 1. Standardized mean differences of the prevalence of topics and LIWC categories from pre- to midpandemic threads. ED: eating disorder; LIWC: Linguistic Inquiry and Word Count.
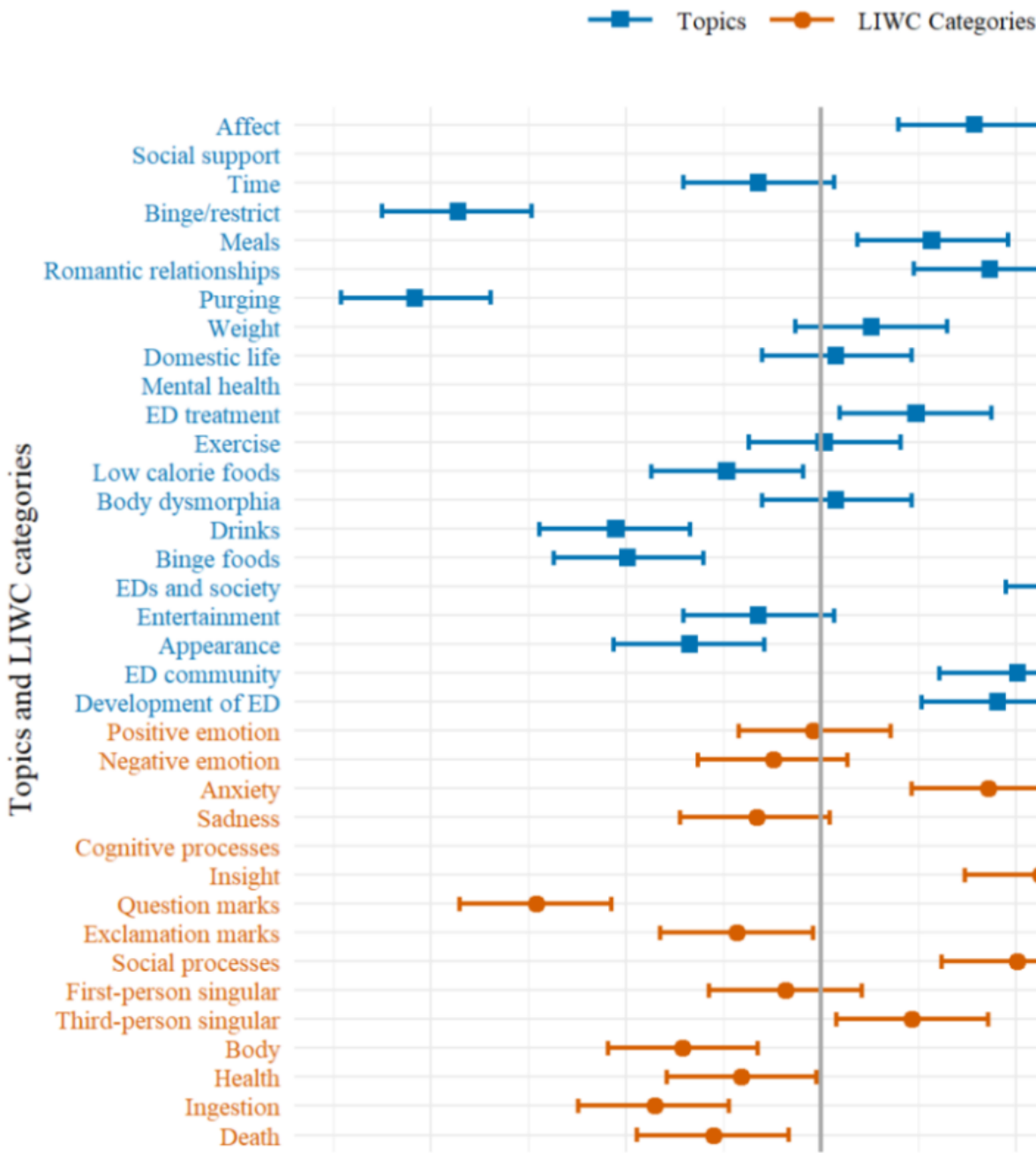

Death

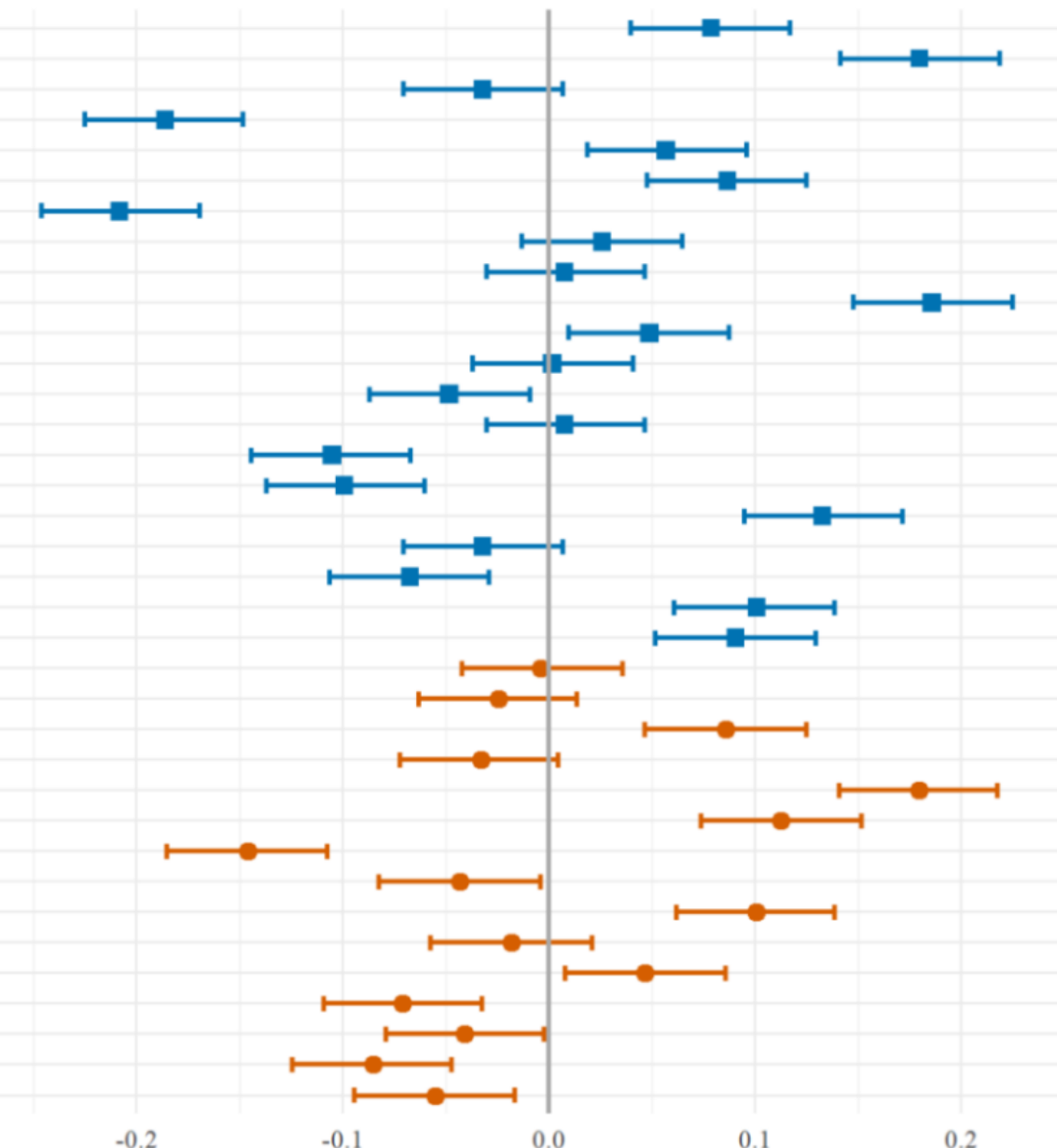

Standardized mean differences from pre- to midpandemic threads

\section{Discussion}

\section{Principal Findings}

This study is the first to explore discussions in one of the largest ED online communities on the social media website Reddit during the onset of the global COVID-19 pandemic. We used automated text analysis methods to investigate changes in language style and topics in comment threads around the time when COVID-19 was declared a global pandemic on March 11,2020 . We were able to identify 21 topics in comment threads using LDA, addressing a number of domains and behaviors of individuals who were actively contributing to the online ED community. They cover areas such as ED symptoms; weight, shape, and eating concerns; mental health and treatment; everyday social life; or the expression of emotions. Additionally, we investigated changes in language styles at the beginning of the global pandemic using a set of indicators covering affective, cognitive, social, and behavioral dimensions of language use.

As the two text analysis methods used in our study follow different approaches, bottom-up in the case of LDA and top-down in the case of LIWC, their combined results provide a more complete picture of discussion in the ED community than either method on its own. By providing unique insights into the online social media behaviors in this large community, our results complement and contrast with existing survey studies on the effects of the pandemic on individuals with EDs (eg, $[12,13])$ and a qualitative analysis of other ED communities on Reddit [28]. With this study, we followed the call to study the experiences of individuals with or at risk of EDs during the global COVID-19 pandemic, as they can aid in developing studies and interventions that address the needs of these vulnerable groups in future crises [14]. 
Although the focus of our study was not on individual users but rather on the community as a whole, our findings were still in line with another study that showed that individuals with EDs were more active on social media during the first months of the COVID-19 pandemic [11]. Recurring users, that is, users who were active in both time periods of our study, had higher participation rates in threads in the midpandemic period. Additionally, a high number of new users joined and participated in the ED community in the midpandemic period. However, the actual number of new individuals in the ED community is most likely lower, as many Reddit users abandon their existing accounts after some time and create new accounts.

Topics covering ED symptom-related discussion such as binge or restrict, purging, low calorie foods, or binge foods had lower prevalences in midpandemic than in prepandemic threads. This was supported by a decrease in the LIWC category ingestion after March 11, 2020, which suggests that the communication in the community focused less frequently on $\mathrm{ED}$ symptom-related discussions at the onset of the global COVID-19 pandemic. This pattern stands in contrast to recent surveys that found marked increases in self-reported ED symptoms during the COVID-19 pandemic $[9,11-13,16]$. However, a qualitative study of ED communities during this time also noted a reduction in ED symptoms in a small percentage of users [28].

At the onset of the COVID-19 pandemic, traditional and social media were focusing on reports about food shortages, weight gain during lockdowns, and home exercises. However, this media attention did not lead to an increase in discussion about topics related to weight, exercise, and body dysmorphia in the ED community, possibly because users already show a high level of preoccupation with these topics. It is striking that communicating about physical exercise has not become more common, as surveys with individuals with an ED indicate an increase in exercising during the pandemic [11]. However, we observed a significant decrease in the prevalence of the appearance topic, which could indicate that users worry less about how they look at this time, most likely because they are staying at home more where they will be seen by fewer people.

Mental health and treatment-related topics became more prevalent in the ED community after the beginning of the pandemic. In their discussions, users might have used social media to address their experiences and concerns with treatment disruptions or the transition of health care services to telehealth applications $[9,11,13]$. However, it could also reflect a genuine effort by users to learn about ED treatments or exchange views on recovery from EDs. A willingness among users of ED communities on Reddit to foster healthier habits and attempt recovery from EDs during the first months of the pandemic was also noted in another study [28]. The increase in discussions could have also been caused by users debating or inquiring about alternatives for their interrupted ED treatments such as self-management of recovery or anonymous helplines $[9,17]$.

On a cognitive level of language, we found a rise in indicators that were associated with cognitive processing, that is, the LIWC categories cognitive processes and insight, in threads. Cognitive processing was previously found to be elevated in blogs advocating recovery from EDs (pro-recovery) compared to pro-ED blogs [39]. This was attributed to individuals in stages of recovery showing greater cognitive reflection or reappraisal of their condition in their blogs. In line with these findings, we also observed an increased language complexity through the decreased use of exclamation marks.

Two topics demonstrating higher levels of cognitive processing were also featured more frequently in midpandemic comment threads. The topic EDs and society subsumes discussions on how EDs and individuals with lived experience of EDs are perceived by society as indicated by words such as culture, shame, or judge. With the topic development of ED, users reflected on events or persons in their past that they believe to have influenced the onset and development of their ED behaviors and thoughts. These discussions required a higher level of abstraction and recollection from their participants.

Users' social life seemed to play a bigger role in the community, as increases in the topic romantic relationships, the LIWC category social words, and in personal pronouns referring to another person (third-person singular) showed. Due to lockdowns, stay-at-home orders, and similar measures, users might be spending more time at home with their partners and families. This can be challenging for individuals with EDs, as they might feel pressured to follow a diet set by others or stressed because they are hiding their ED behaviors from others [11]. Users might be venting about social interactions or conflicts with others in their household using words from the aforementioned categories and topics. Shared meals represent another source for potential conflicts, as others can observe what and how much the individual with an ED is eating, which could have led to the increase in discussions about meals [11]. The amount of social references differentiated between pro-recovery and pro-ED content in a previous study, with less social references found in pro-ED texts and more social activity being associated with recovery [39]. This is attributed to a withdrawal from others or avoidance of connections with others typical for EDs. The higher frequency of social references in our study indicates that users are either having more real-world interactions about which they reflect in the online community or, alternatively, that more of their social life is happening in the community because real-life social relationships were restricted, both supporting a positive trend away from ED-typical social withdrawal.

The rise in frequency of anxiety-related words could be an indicator for elevated health anxiety and worries about contracting SARS-CoV-2 $[9,10]$. Indeed, health anxiety has emerged across a number of mental health communities including the ED community investigated in this study in the wake of the pandemic [41]. Additionally, anxiety about COVID-19 was related to higher ED pathology in one study [42]. In this context, it is surprising that we found a decrease in prevalence of the LIWC categories assessing health and death, as these encompass words that could be used to discuss COVID-19, its treatment, and sequelae.

Although it might appear contradictory to observe a rise in the topic affect without a corresponding increase in neither the positive emotions nor negative emotions categories, this 
discrepancy can be explained by the composition of each category. The affect topic captured words representing emotions, such as shame, guilt, or anger, which are, however, featured to a much lower degree in the positive emotions or negative emotions dictionaries.

The increase in prevalence of anxiety words reflects the expression of fears associated with the serious consequences of COVID-19; however, it also points to a rise in emotional awareness and disclosure that would be more typical of recovery texts [39]. A stronger inhibition of negative emotions resulting in lower expression of negative emotion words would be expected in individuals affected by EDs rather than in individuals currently recovering from an ED. However, we did not observe any significant reductions in words from the LIWC category sadness in midpandemic threads.

In sum, our results illustrate pronounced changes in the online community that suggest a perspective shift in the discussions in the ED community from a narrow focus on ED symptoms and potentially harmful weight loss methods toward a broader perspective with increased attention to mental health, social resources, and treatments for EDs. This shift is characterized by heightened cognitive processing, more social references, less inhibition of negative emotions, and discussions focused less on ED symptoms and more on mental health and treatment. It is important to note here that the rules set by the community's moderators claim to not explicitly identify as either being pro-recovery or pro-ED, as the community would offer support to all and would encourage or promote neither ED behaviors nor recovery from EDs (quote paraphrased to protect the anonymity of the ED community). Although some social media platforms have distinct pro-recovery and pro-ED communities, which show seemingly little interaction between their members [43], other online ED communities feature pro-recovery and pro-ED content alongside each other [44]. It is therefore possible that the communication pattern observed in an ED community might move toward the pro-recovery end of a theoretical spectrum between pro-ED and pro-recovery. It is yet unclear whether this is a sustained change or a temporary phenomenon pushed by the disruptive situation of the recent pandemic. We also cannot deduce from these results whether they will lead to many users attempting or achieving recovery from EDs, as this can be a protracted and difficult process that is often accompanied by relapses. Remaining active in online ED communities could make achieving recovery even more difficult [44].

Other salient results from this study concern factors such as social support and the ED community. Expressions of social support became more frequent at the beginning of the pandemic. However, social support in online ED communities can be a double-edged sword, as many users report that they value the communities for the support they provide [26], while researchers suggest that support is often tied to following group norms that foster potentially harmful ED behaviors and thoughts [20]. Therefore, supportive words might be extended to users directly affected by the pandemic, to those seeking help with their condition, and potentially to those wanting to lose weight using potentially harmful methods. The topic ED community contains references to the ED community and Reddit in general. It could represent a kind of meta-discussion about the community and its place among other communities on Reddit with the topic. Its significant increase might reflect a kind of growth in awareness of the community and how it can support its users. These potential consequences of the pandemic on the structure of social media warrant further exploration in future research.

\section{Limitations}

This study is limited by the fact that we do not know what the relation between discussions in an ED community and real-life behaviors is. Although we found decreases in ED symptom-related discussions and increases in discussions about social and mental health issues, we do not know whether these changes are accompanied by reductions in ED behaviors and increased help-seeking or treatment uptake. However, the shift in discussion points to the community itself becoming a place that is more open to treatment and recovery from EDs.

A second limitation is that the ED community and Reddit as a whole can be used anonymously, precluding us from making inferences on the demographics or ED impairment of users in the community. Additionally, we cannot draw conclusions on changes due to the pandemic for particular users or user groups, as we were interested in changes in the community as a whole and thus estimated prevalences of language style and topics at the level of threads. As our analysis methods treat these threads, which combine a post and comments from different users as bags of words, we were unable to trace back the prevalence of topics and LIWC categories or their changes to specific users or groups of users. Although it is possible that the same users went from discussing ED symptoms to asking about mental health and treatment, it could also have been entirely new users that changed the discussion.

A third limitation concerns the time period under study. We observed the online ED community in the first few months of the pandemic, and it is yet unclear whether the observed changes will endure over time. Additionally, we cannot ascertain whether the changes we observed are fully due to the COVID-19 pandemic or whether seasonal effects also play a role. Plots of mean daily prevalence rates of topics and LIWC categories over the whole time span of our study in Supplement S3 and S4 in Multimedia Appendix 1 can give some indication whether effects are due to short spikes or more enduring trends.

\section{Conclusions}

The COVID-19 pandemic and its accompanying public health measures have disrupted the everyday life of people worldwide, possibly impacting their psychological well-being and coping strategies. Clinical researchers are understandably concerned about how these changes affect individuals with mental disorders. We present in this study a snapshot of changes in an online ED community during the first few months of the pandemic. As such, we aim to contribute to the growing area of research on the experiences of individuals affected by disordered eating during this time. Our specific contribution lies in categorizing the natural discussions occurring in the ED community on Reddit into content and language style categories and uncovering how discussions changed at the beginning of the global pandemic. The presented results suggest that reaching 
out to users of online ED communities and recruiting them for treatment interventions might be especially effective at this time as need, openness, and interest for mental health treatment increases. Additionally, the language in discussions changed in a way that suggests a move toward a stronger focus on recovery and mental health treatment in the community. The changes we observed reflect issues users were experiencing in real life during the first wave of the COVID-19 pandemic. Understanding these issues can aid in developing interventions that can mitigate the consequences of future waves of the COVID-19 pandemic or other similar disease outbreaks in the future for individuals with EDs.

\section{Acknowledgments}

Preparation of this manuscript was supported by the Digital Society Initiative of the University of Zurich. The authors acknowledge financial support by the Open Access Publishing Fund of Ruprecht-Karls-Universität Heidelberg.

\section{Conflicts of Interest}

None declared.

\section{Multimedia Appendix 1}

Supplementary material.

[DOCX File, 1074 KB-Multimedia Appendix 1]

\section{References}

1. Vindegaard N, Benros ME. COVID-19 pandemic and mental health consequences: systematic review of the current evidence. Brain Behav Immun 2020 Oct;89:531-542 [FREE Full text] [doi: 10.1016/j.bbi.2020.05.048] [Medline: $\underline{32485289}$ ]

2. Luo M, Guo L, Yu M, Jiang W, Wang H. The psychological and mental impact of coronavirus disease 2019 (COVID-19) on medical staff and general public - A systematic review and meta-analysis. Psychiatry Res 2020 Sep;291:113190 [FREE Full text] [doi: 10.1016/j.psychres.2020.113190] [Medline: 32563745]

3. Neelam K, Duddu V, Anyim N, Neelam J, Lewis S. Pandemics and pre-existing mental illness: a systematic review and meta-analysis. Brain Behav Immun Health 2021 Jan;10:100177 [FREE Full text] [doi: 10.1016/j.bbih.2020.100177] [Medline: $\underline{3251527]}$

4. Brooks SK, Webster RK, Smith LE, Woodland L, Wessely S, Greenberg N, et al. The psychological impact of quarantine and how to reduce it: rapid review of the evidence. Lancet 2020 Mar 14;395(10227):912-920 [FREE Full text] [doi: 10.1016/S0140-6736(20)30460-8] [Medline: 32112714]

5. Benke C, Autenrieth LK, Asselmann E, Pané-Farré CA. Lockdown, quarantine measures, and social distancing: associations with depression, anxiety and distress at the beginning of the COVID-19 pandemic among adults from Germany. Psychiatry Res 2020 Nov;293:113462 [FREE Full text] [doi: 10.1016/j.psychres.2020.113462] [Medline: 32987222]

6. Gan Y, Ma J, Wu J, Chen Y, Zhu H, Hall BJ. Immediate and delayed psychological effects of province-wide lockdown and personal quarantine during the COVID-19 outbreak in China. Psychol Med 2020 Aug 13:1-12 [FREE Full text] [doi: 10.1017/S0033291720003116] [Medline: 32787981]

7. Liu X, Kakade M, Fuller CJ, Fan B, Fang Y, Kong J, et al. Depression after exposure to stressful events: lessons learned from the severe acute respiratory syndrome epidemic. Compr Psychiatry 2012 Jan;53(1):15-23 [FREE Full text] [doi: 10.1016/j.comppsych.2011.02.003] [Medline: 21489421]

8. Jeong H, Yim HW, Song Y, Ki M, Min J, Cho J, et al. Mental health status of people isolated due to Middle East respiratory syndrome. Epidemiol Health 2016;38:e2016048. [doi: 10.4178/epih.e2016048] [Medline: 28196409]

9. Clark Bryan D, Macdonald P, Ambwani S, Cardi V, Rowlands K, Willmott D, et al. Exploring the ways in which COVID-19 and lockdown has affected the lives of adult patients with anorexia nervosa and their carers. Eur Eat Disord Rev 2020 Nov;28(6):826-835 [FREE Full text] [doi: 10.1002/erv.2762] [Medline: 32643844]

10. Termorshuizen JD, Watson HJ, Thornton LM, Borg S, Flatt RE, MacDermod CM, et al. Early impact of COVID-19 on individuals with self-reported eating disorders: a survey of $\sim 1,000$ individuals in the United States and the Netherlands. Int J Eat Disord 2020 Nov;53(11):1780-1790. [doi: 10.1002/eat.23353] [Medline: 32720399]

11. Branley-Bell D, Talbot C. Exploring the impact of the COVID-19 pandemic and UK lockdown on individuals with experience of eating disorders. J Eat Disord 2020;8:44 [FREE Full text] [doi: 10.1186/s40337-020-00319-y] [Medline: 32874585]

12. Phillipou A, Meyer D, Neill E, Tan EJ, Toh WL, Van Rheenen TE, et al. Eating and exercise behaviors in eating disorders and the general population during the COVID-19 pandemic in Australia: initial results from the COLLATE project. Int $\mathbf{J}$ Eat Disord 2020 Jul;53(7):1158-1165 [FREE Full text] [doi: 10.1002/eat.23317] [Medline: 32476163]

13. Schlegl S, Meule A, Favreau M, Voderholzer U. Bulimia nervosa in times of the COVID-19 pandemic-results from an online survey of former inpatients. Eur Eat Disord Rev 2020 Nov;28(6):847-854 [FREE Full text] [doi: 10.1002/erv.2773] [Medline: 32767858]

14. Weissman RS, Bauer S, Thomas JJ. Access to evidence-based care for eating disorders during the COVID-19 crisis. Int J Eat Disord 2020 May;53(5):369-376 [FREE Full text] [doi: 10.1002/eat.23279] [Medline: $\underline{\text { 32338400] }}$ 
15. Machado PPP, Pinto-Bastos A, Ramos R, Rodrigues TF, Louro E, Gonçalves S, et al. Impact of COVID-19 lockdown measures on a cohort of eating disorders patients. J Eat Disord 2020 Nov 02;8(1):57 [FREE Full text] [doi: 10.1186/s40337-020-00340-1] [Medline: 33292539]

16. Schlegl S, Maier J, Meule A, Voderholzer U. Eating disorders in times of the COVID-19 pandemic-results from an online survey of patients with anorexia nervosa. Int J Eat Disord 2020 Nov;53(11):1791-1800 [FREE Full text] [doi: 10.1002/eat.23374] [Medline: 32841413]

17. Richardson C, Patton M, Phillips S, Paslakis G. The impact of the COVID-19 pandemic on help-seeking behaviors in individuals suffering from eating disorders and their caregivers. Gen Hosp Psychiatry 2020;67:136-140. [doi: 10.1016/j.genhosppsych.2020.10.006] [Medline: 33129138]

18. Pearl RL. Weight stigma and the "Quarantine-15". Obesity (Silver Spring) 2020 Jul;28(7):1180-1181 [FREE Full text] [doi: 10.1002/oby.22850] [Medline: 32324954]

19. Rains SA, Peterson EB, Wright KB. Communicating social support in computer-mediated contexts: a meta-analytic review of content analyses examining support messages shared online among individuals coping with illness. Commun Monogr 2015 Mar 17;82(4):403-430. [doi: 10.1080/03637751.2015.1019530]

20. Rouleau CR, von Ranson KM. Potential risks of pro-eating disorder websites. Clin Psychol Rev 2011 Jun;31(4):525-531. [doi: 10.1016/j.cpr.2010.12.005] [Medline: 21272967]

21. Harper K, Sperry S, Thompson JK. Viewership of pro-eating disorder websites: association with body image and eating disturbances. Int J Eat Disord 2008 Jan;41(1):92-95. [doi: 10.1002/eat.20408] [Medline: 17634964]

22. Borzekowski DLG, Schenk S, Wilson JL, Peebles R. e-Ana and e-Mia: a content analysis of pro-eating disorder Web sites. Am J Public Health 2010 Aug;100(8):1526-1534. [doi: 10.2105/AJPH.2009.172700] [Medline: 20558807]

23. Juarascio AS, Shoaib A, Timko CA. Pro-eating disorder communities on social networking sites: a content analysis. Eat Disord 2010;18(5):393-407. [doi: 10.1080/10640266.2010.511918] [Medline: 20865593]

24. Peebles R, Wilson JL, Litt IF, Hardy KK, Lock JD, Mann JR, et al. Disordered eating in a digital age: eating behaviors, health, and quality of life in users of websites with pro-eating disorder content. J Med Internet Res 2012 Oct 25;14(5):e148 [FREE Full text] [doi: 10.2196/jmir.2023] [Medline: 23099628]

25. Rodgers RF, Lowy AS, Halperin DM, Franko DL. A meta-analysis examining the influence of pro-eating disorder websites on body image and eating pathology. Eur Eat Disord Rev 2016 Jan;24(1):3-8. [doi: 10.1002/erv.2390] [Medline: 26230192]

26. Csipke E, Horne O. Pro-eating disorder websites: users' opinions. Eur Eat Disord Rev 2007 May;15(3):196-206. [doi: 10.1002/erv.789] [Medline: 17676689]

27. Sowles SJ, McLeary M, Optican A, Cahn E, Krauss MJ, Fitzsimmons-Craft EE, et al. A content analysis of an online pro-eating disorder community on Reddit. Body Image 2018 Mar;24:137-144 [FREE Full text] [doi: 10.1016/j.bodyim.2018.01.001] [Medline: 29414146]

28. Nutley SK, Falise AM, Henderson R, Apostolou V, Mathews CA, Striley CW. Impact of the COVID-19 pandemic on disordered eating behavior: qualitative analysis of social media posts. JMIR Ment Health 2021 Jan 27;8(1):e26011 [FREE Full text] [doi: 10.2196/26011] [Medline: $\underline{\text { 33465035] }}$

29. Al-Garadi MA, Khan MS, Varathan KD, Mujtaba G, Al-Kabsi AM. Using online social networks to track a pandemic: a systematic review. J Biomed Inform 2016 Aug;62:1-11 [FREE Full text] [doi: 10.1016/j.jbi.2016.05.005] [Medline: $\underline{27224846}]$

30. Wang Y, McKee M, Torbica A, Stuckler D. Systematic literature review on the spread of health-related misinformation on social media. Soc Sci Med 2019 Nov;240:112552 [FREE Full text] [doi: 10.1016/j.socscimed.2019.112552] [Medline: $\underline{31561111]}$

31. Murray C, Mitchell L, Tuke J, Mackay M. Symptom extraction from the narratives of personal experiences with COVID-19 on Reddit. arXiv Preprint posted online on May 21, 2020.

32. Su Y, Xue J, Liu X, Wu P, Chen J, Chen C, et al. Examining the impact of COVID-19 lockdown in Wuhan and Lombardy: a psycholinguistic analysis on Weibo and Twitter. Int J Environ Res Public Health 2020 Jun 24;17(12):4552 [FREE Full text] [doi: 10.3390/ijerph17124552] [Medline: 32599811]

33. Listings of WHO's response to COVID-19. World Health Organization. 2020. URL: https://www.who.int/news-room/ detail/29-06-2020-covidtimeline [accessed 2021-06-11]

34. Moessner M, Feldhege J, Wolf M, Bauer S. Analyzing big data in social media: text and network analyses of an eating disorder forum. Int J Eat Disord 2018 Jul;51(7):656-667. [doi: 10.1002/eat.22878] [Medline: 29746710]

35. Blei DM, Ng AY, Jordan MI. Latent Dirichlet allocation. J Machine Learning Res 2000:993-1022 [FREE Full text]

36. Feldhege J, Moessner M, Bauer S. Who says what? Content and participation characteristics in an online depression community. J Affect Disord 2020 Feb 15;263:521-527. [doi: 10.1016/j.jad.2019.11.007] [Medline: $\underline{31780138]}$

37. Airoldi EM, Bischof JM. Improving and evaluating topic models and other models of text. J Am Stat Assoc 2017 Jan 04;111(516):1381-1403. [doi: 10.1080/01621459.2015.1051182]

38. Mimno D, Wallach HM, Talley E, Leenders M, McCallum A. Optimizing semantic coherence in topic models. 2011 Presented at: Conference on Empirical Methods in Natural Language Processing; July 27-31, 2011; Edinburgh, Scotland, UK p. 262-272. 
39. Wolf M, Theis F, Kordy H. Language use in eating disorder blogs. J Lang Soc Psychol 2013 Feb 07;32(2):212-226. [doi: 10.1177/0261927X12474278]

40. Pennebaker JW, Boyd RL, Jordan K, Blackburn K. The Development and Psychometric Properties of LIWC2015. Austin, TX: University of Texas at Austin; 2015.

41. Low DM, Rumker L, Talkar T, Torous J, Cecchi G, Ghosh SS. Natural language processing reveals vulnerable mental health support groups and heightened health anxiety on Reddit during COVID-19: observational study. J Med Internet Res 2020 Oct 12;22(10):e22635 [FREE Full text] [doi: 10.2196/22635] [Medline: 32936777]

42. Scharmer C, Martinez K, Gorrell S, Reilly EE, Donahue JM, Anderson DA. Eating disorder pathology and compulsive exercise during the COVID-19 public health emergency: examining risk associated with COVID-19 anxiety and intolerance of uncertainty. Int J Eat Disord 2020 Dec;53(12):2049-2054. [doi: 10.1002/eat.23395] [Medline: 33098579]

43. Yom-Tov E, Fernandez-Luque L, Weber I, Crain SP. Pro-anorexia and pro-recovery photo sharing: a tale of two warring tribes. J Med Internet Res 2012 Nov 07;14(6):e151 [FREE Full text] [doi: 10.2196/jmir.2239] [Medline: 23134671]

44. Chancellor S, Mitra T, De Choudhury M. Recovery amid pro-anorexia: analysis of recovery in social media. Proc SIGCHI Conf Hum Factor Comput Syst 2016 May;2016:2111-2123 [FREE Full text] [doi: 10.1145/2858036.2858246] [Medline: 28840201]

\title{
Abbreviations \\ API: application programming interface \\ ED: eating disorder \\ LDA: latent Dirichlet allocation \\ LIWC: Linguistic Inquiry and Word Count \\ SMD: standardized mean difference
}

\author{
Edited by C Basch; submitted 02.03.21; peer-reviewed by C Oehler, J Chen, S Chancellor; comments to author 13.04.21; revised \\ version received 11.05.21; accepted 18.05.21; published 08.07.21 \\ Please cite as: \\ Feldhege J, Moessner M, Wolf M, Bauer S \\ Changes in Language Style and Topics in an Online Eating Disorder Community at the Beginning of the COVID-19 Pandemic: \\ Observational Study \\ J Med Internet Res 2021;23(7):e28346 \\ URL: https://www.jmir.org/2021/7/e28346 \\ doi: $10.2196 / 28346$ \\ PMID: 34101612
}

(CJohannes Feldhege, Markus Moessner, Markus Wolf, Stephanie Bauer. Originally published in the Journal of Medical Internet Research (https://www.jmir.org), 08.07.2021. This is an open-access article distributed under the terms of the Creative Commons Attribution License (https://creativecommons.org/licenses/by/4.0/), which permits unrestricted use, distribution, and reproduction in any medium, provided the original work, first published in the Journal of Medical Internet Research, is properly cited. The complete bibliographic information, a link to the original publication on https://www.jmir.org/, as well as this copyright and license information must be included. 